\title{
Plant and soil biodiversity have non-substitutable stabilizing effects on biomass production
}

\author{
Gaowen Yang ${ }^{1}$, Masahiro Ryo $^{1}$, Julien Roy ${ }^{1}$, Stefan Hempel $^{1}$, and Matthias Rillig ${ }^{2}$ \\ ${ }^{1}$ Freie Universität Berlin \\ ${ }^{2}$ Freie Universitaet Berlin
}

January 12, 2021

\begin{abstract}
The stability of plant biomass production in the face of environmental change is fundamental for maintaining terrestrial ecosystem functioning, as plant biomass is the ultimate source of energy for nearly all life forms. However, most studies have focused on the stabilizing effect of plant diversity, neglecting the effect of soil biodiversity, the largest reservoirs of biodiversity on Earth. Here we investigated the effects of plant and soil biodiversity on the temporal stability of biomass production under varying simulated precipitation in grassland microcosms. Soil biodiversity loss reduced temporal stability by suppressing asynchronous responses of plant functional groups. Greater plant diversity, especially in terms of functional diversity, promoted temporal stability, but this effect was independent of soil biodiversity loss. Moreover, multitrophic biodiversity, plant and soil biodiversity combined, was positively associated with temporal stability. Our study highlights the importance of maintaining the biodiversity of multiple trophic levels for sustainable biomass production.
\end{abstract}

Plant and soil biodiversity have non-substitutable stabilizing effects on biomass production Gaowen Yang ${ }^{1,2^{*}}$, Masahiro Ryo ${ }^{1,2,3,4}$, Julien Roy ${ }^{1,2}$, Stefan Hempel ${ }^{1,2}$, Matthias C. Rillig ${ }^{1,2}$

${ }^{1}$ Institut für Biologie, Freie Universität Berlin, D-14195 Berlin, Germany

${ }^{2}$ Berlin-Brandenburg Institute of Advanced Biodiversity Research (BBIB), D-14195 Berlin, Germany

${ }^{3}$ Leibniz Centre for Agricultural Landscape Research (ZALF), Eberswalder Str. 84, 15374 Müncheberg, Germany

${ }^{4}$ Environment and Natural Science, Brandenburg University of Technology, Cottbus-Senftenberg (BTU), 03046 Cottbus, Germany

Gaowen Yang,yanggw@zedat.fu-berlin.de

Masahiro Ryo,masahiroryo@gmail.com

Julien Roy,royjulien@zedat.fu-berlin.de

Stefan Hempel,stefan.hempel@fu-berlin.de

Matthias C. Rillig,rillig@zedat.fu-berlin.de

Short running title : Plant and soil biodiversity affect stability

Keywords : biodiversity loss, community-weighted mean, drought, dilution-to-extinction approach, ecosystem stability, functional diversity, multitrophic biodiversity, species asynchrony, temporal stability

Type of article : Letters 


\section{Word count :}

The number of words in the abstract:

The number of words in the main text (excluding abstract, acknowledgements, references, table and figure legends):

The number of references:

4959

The number of words in each text box:

The number of figures, tables, and text boxes:

The number of tables:

The number of text boxes:

${ }^{*}$ Corresponding author : Gaowen Yang

Tel: +493083853172

Email:yanggw@zedat.fu-berlin.de

Statement of authorship : GY and MCR conceived the ideas and designed the study. GY set up the experiment and collected the data, with important suggestions from SH. MR, JR and GY analyzed the data. GY, MR and JR wrote the first draft, and all authors commented on the manuscript.

Data accessibility statement: We confirm that, should the manuscript be accepted, the data supporting the results will be archived in an appropriate public repository (Dryad, Figshare or Hal) and the data DOI will be included at the end of the article.

Abstract ( $<150$ words)

The stability of plant biomass production in the face of environmental change is fundamental for maintaining terrestrial ecosystem functioning, as plant biomass is the ultimate source of energy for nearly all life forms. However, most studies have focused on the stabilizing effect of plant diversity, neglecting the effect of soil biodiversity, the largest reservoirs of biodiversity on Earth. Here we investigated the effects of plant and soil biodiversity on the temporal stability of biomass production under varying simulated precipitation in grassland microcosms. Soil biodiversity loss reduced temporal stability by suppressing asynchronous responses of plant functional groups. Greater plant diversity, especially in terms of functional diversity, promoted temporal stability, but this effect was independent of soil biodiversity loss. Moreover, multitrophic biodiversity, plant and soil biodiversity combined, was positively associated with temporal stability. Our study highlights the importance of maintaining the biodiversity of multiple trophic levels for sustainable biomass production.

\section{Introduction}

Understanding the mechanisms that stabilize ecosystem functions when faced with a changing environment has been a key issue in ecology (Tilman \& Downing 1994; Tilman et al. 2006; Isbell et al.2009; Hector et al. 2010; de Mazancourt et al. 2013; Loreau \& de Mazancourt 2013; Hautier et al. 2014; Cravenet al. 2018; Wang et al. 2019b). Greater biodiversity, especially plant diversity, usually increases the temporal stability of biomass production, although negative and neutral effects have been occasionally observed (Tilman et al. 2006; Hector et al. 2010; Hautier et al. 2014; Pennekamp et al. 2018; Craven et al.2018; Wang et al. 2019b). However, the vast majority of existing studies focused on the biodiversity of a single trophic group (Tilmanet al. 2006; Isbell et al. 2009; Hector et al.2010; Hautier et al. 2014; Hautier et al. 2015; Maet al. 2017; Wang et al. 2019b), neglecting biodiversity across trophic levels, even though multitrophic biodiversity has been shown to drive multiple ecosystem functions (Soliveres et al.2016; Schuldt et al. 2018; Geisen et al. 2019; Domeignoz-Horta et al. 2020).

Soil biota, comprising an enormous number of consumers and decomposers, represent one of the largest reservoirs of biodiversity on Earth (Orgiazzi et al.2016; Geisen et al. 2019; Thakur et al.2020). A growing number of studies suggest that soil biodiversity has an essential impact on plant diversity, community composition, biomass production, plant-plant interactions and plant tolerance to stress factors, as well as nutrient cycling 
(Wagg et al. 2014; Bardgett \& van der Putten 2014; Delgado-Baquerizo et al. 2016; Wagg et al. 2019; Liang et al. 2019; Domeignoz-Horta et al. 2020; Guerra et al. 2020). Recent work has provided a conceptual framework showing that plant and soil biodiversity can jointly influence the stability of biomass production via regulating these plant attributes (Yang et al. 2018). However, there is increasing concern that soil and plant biodiversity is threatened by anthropogenic environmental change (Tsiafouli et al. 2015; Gossner et al. 2016; Banerjeeet al. 2019; Geisen et al. 2019; Zhou et al. 2020). Hence, it is important to understand how biodiversity loss in both soil and plant communities affects the stability of biomass production will provide a broader perspective.

Here we conducted a fully factorial experiment manipulating plant diversity and soil biodiversity using model grassland microcosms (Fig. 1). We used a dilution-to-extinction approach (Yan et al. 2015; Hol et al. 2015; Roger et al. 2016) to create a gradient of soil biodiversity, and then established grassland microcosms of different plant species richness at each point along the soil biodiversity gradient under greenhouse conditions. We focused on the temporal stability of biomass production, defined as the ratio of the temporal mean to the standard deviation of plant community biomass (Tilmanet al. 2006). Because the constant environment in the greenhouse does not capture natural variability, environmental variation in precipitation was simulated by inducing three wet-dry cycles in all microcosms to investigate the effects of the biodiversity treatments on the temporal stability of biomass production.

Considering the significance of soil biodiversity on plant attributes (Yang et al. 2018), we predicted that soil biodiversity loss will reduce the temporal stability of biomass production. Specifically, we tested whether and how soil biodiversity interacts with plant diversity in terms of species richness and functional diversity to influence temporal stability. Furthermore, we investigated how multitrophic biodiversity, accounting for plant and soil biodiversity, affects temporal stability. We find that both greater plant and soil biodiversity had positive effects on the temporal stability of community biomass production, but plant and soil biodiversity independently affected temporal stability. Multitrophic biodiversity is positively associated with temporal stability.

\section{Material and methods}

Experimental design. This experiment was set up as a fully factorial design containing all combinations of three levels of soil biodiversity (low, moderate, high) and four levels of plant species richness (1, 4, 8, 12 species) (Fig. 1 and Supplementary Methods, Table S1). We created the four- and eight- plant species treatments, which were replicated 7 times, by randomly selecting plant species from a 12-species pool for each replicate. For each replicate of plant diversity treatments, there were identical species composition among low, moderate and high soil biodiversity treatments to avoid a confounding effect of plant community composition and soil biodiversity treatments. There were 12 treatments and a total of 123 grassland microcosms. Twelve typical species from a local grassland in Brandenburg, Germany, were used in this study: four grasses (Holcus lanatus, Anthoxanthum odoratum, Lolium perenne and Festuca rubra), four herbs (Daucus carota ,Achillea millefolium, Hieracium pilosella andPlantago lanceolata) and four legumes (Trifolium repens, Vicia cracca , Medicago lupulina and Lotus corniculatus ).

Soil inoculum preparation. We used the dilution-to-extinction approach (Yan et al. 2015; Hol et al. 2015; Roger et al. 2016; Maron et al. 2018; Domeignoz-Horta et al. 2020) to create high, moderate and low soil biodiversity (Fig. 1 and Supplementary Methods). Fresh field soil was collected from the top $20 \mathrm{~cm}$ of the local grassland in Brandenburg (Rillig et al. 2010). The fresh field soil was treated as the undiluted treatment $\left(10^{\circ}\right)$. We mixed $200 \mathrm{~g}$ dw (dry weight) of fresh soil with $1800 \mathrm{~g}$ dw sterilized soil (autoclaving for $90 \mathrm{~min}$ at $121^{\circ} \mathrm{C}$ ) to obtain the $10^{-1}$ dilution treatment, and the $200 \mathrm{~g}$ of the $10^{-1}$ dilution was mixed with $1800 \mathrm{~g} \mathrm{dw}$ sterilized soil to obtain the $10^{-2}$ dilution treatment. This procedure was repeated several times to reach $10^{-3}$ and $10^{-6}$ dilutions. A plastic bag was filled with $2000 \mathrm{~g} \mathrm{dw}$ of the $10^{0}, 10^{-3}$ or $10^{-6}$ dilutions, and then received sterile water to reach the initial soil moisture. To recover soil microbial biomass, all soil dilutions were incubated in a dark room $\left(20^{\circ} \mathrm{C}\right)$ for two months, until no differences among soil dilutions were observed using the substrate-induced respiration method (Fig. 2a). The $10^{0}, 10^{-3}$ and $10^{-6}$ dilutions of soil inocula were treated as high, moderate and low soil biodiversity treatments, respectively. 
Microcosms establishment and sampling. Each grassland microcosm $(22.5 \mathrm{~cm}$ diameter and $16.5 \mathrm{~cm}$ height) was established by filling with $5.5 \mathrm{~L}(6.8 \mathrm{~kg})$ of a sterilized 1:1 sand:field soil mixture (autoclaving for $90 \mathrm{~min}$ at $121^{\circ} \mathrm{C}$ ) and $200 \mathrm{~g}$ of soil inoculum. Microcosm with 1, 4, 8 or 12 plant species richness treatment received 24, 6, 3 or 2 seedlings of each species, respectively. All microcosms were maintained in a climatecontrolled greenhouse.

We simulated environmental variation in precipitation by inducing three wet-dry cycles (Fig. 1). Each microcosm was watered twice weekly to maintain gravimetric soil moisture of $12-18 \%$ by weight during the wet periods. During the dry periods, each microcosm received $300 \mathrm{~mL}$ of water only when most plant species started to wilt. Each of wet and dry periods lasted at least for 8 weeks. At the end of each period, all plant shoots were harvested by cutting at $5 \mathrm{~cm}$ above the soil surface, sorted by species, oven-dried for $48 \mathrm{~h}$ at $70^{\circ} \mathrm{C}$ and weighed. In total, there were six such harvests, including shoot biomass from 17,712 plant individuals. Based on the maximum nutrients removed by the first harvest, $400 \mathrm{~mL}$ of Hoagland nutrient solution was added to each microcosm after each harvest.

Soil fungal and bacterial diversity. After the final harvest, $100 \mathrm{~g}$ of fresh soil was collected from each of the 12 -species microcosms in each soil biodiversity treatment. Fresh soil samples were stored at $-80^{\circ} \mathrm{C}$ for DNA extraction to evaluate the establishment of the soil microbial community during the experimental period. DNA from each soil inocula and fresh soil samples of the final harvest was extracted from $250 \mathrm{mg}$ soil, using DNeasy PowerMax Soil Kits (MoBio Laboratories Inc., Carlsbad, CA, USA), following manufacturer's instructions. Soil fungal and bacterial diversity were determined following Illumina MiSeq high-throughput sequencing with fITS7 and ITS4 for fungi and 515f and 806r for bacteria (Fierer et al. 2005; Ihrmark et al.2012). Sequencing raw data were processed using DADA2 (Callahan et al. 2016) to obtain denoised, chimera-free, non-singleton amplicon sequence variants (ASVs). Soil microbial diversity was indicated by the sum of bacterial and fungal ASVs in each soil biodiversity treatment. Details of sequence processing can be found in the Supplementary Methods.

Temporal stability of biomass production . The temporal stability of plant community biomass production in each microcosm is defined as $\mu / \sigma$, where $\mu$ is the temporal mean of biomass production during three rounds of wet-dry cycles, and ois the standard deviation of biomass production across this experimental period (Tilman et al. 2006). We partitioned the temporal stability into community-wide plant species asynchrony and weighted population variance (Thibaut \& Connolly 2013). Plant species asynchrony (Loreau \& de Mazancourt 2008) was calculated as $1-\varphi=1-\sigma^{2} /\left(\sum_{i=1}^{s} \sigma_{\mathrm{i}}\right)^{2}$, where $\varphi$ is species synchrony, $\sigma^{2}$ the temporal variance of biomass production, $\sigma_{l}$ is the standard deviation in the shoot biomass of species $i$ in a community with $S$ species. If $1-\varphi$ equals 0 , species fluctuate synchronously, indicating that there is no compensatory effect; if $1-\varphi$ is higher than 0 , species fluctuate asynchronously, indicating that there are compensatory effects (Loreau \& de Mazancourt 2008; Song \& Yu 2015). Asynchrony of plant functional groups was calculated by replacing species i by each plant functional group, which indicates the strength of asynchronous responses at the plant functional group level. Weighted population variance $\left(C V_{\text {pop }}\right)$ is defined as $C V$ pop $=\left(\sum_{i=1}^{s} \sigma_{\mathrm{i}}\right) / \mu$. A decrease in population variance can promote temporal stability by reducing the variability of populations (Thibaut \& Connolly 2013).

Functional composition and diversity . To better understand the role of plant diversity, we calculated four functional diversity indices for each plant community using ten leaf and root traits relevant to drought tolerance: functional richness, functional evenness, functional divergence, and functional dispersion (Villéger et al. 2008; Laliberté \& Legendre 2010; Fischer et al. 2016; Liu et al. 2018; Lozano \& Rillig 2020; Freschet et al. 2020; Mahautet al. 2020) (See Supplementary Methods for details). Principal component analysis (PCA) of community-weighted means of ten traits was employed, using the PCA function in FactoMineR (Lêet al. 2008). The first axis of PCA analysis was treated as an index of the community-weighted mean (CWM) of all traits (Cravenet al. 2018; Mahaut et al. 2020). Functional diversity indices of each harvest were calculated, and then were averaged across the six harvest time points.

Statistical analysis. We used linear models to test the effects of the dilution-to-extinction approach on soil microbial, fungal and bacterial diversity, and microbial biomass. Microbial data were log-transformed 
before analyses to meet the normality assumption. We tested whether soil biodiversity interacts with plant diversity to influence response variables using a linear model. Plant species richness was log-transformed to represent the biodiversity effect which typically saturates with increasing species richness due to increasing redundancy (Hautier et al. 2014). For each response variable, a weighted least squares regression was applied to take heteroskedasticity into account. Since variance differed with plant species richness, each sample was weighted with an inverse of the variance at each plant species richness level. We evaluated the importance of the interaction between soil biodiversity and plant diversity treatments based on model comparison using Akaike information criterion (AIC) that compares the additive model (soil biodiversity + plant species richness) and the interactive model (the additive model with their interaction term).

We calculated multitrophic biodiversity as an average of standardized plant species richness and the number of soil microbial ASVs (Allanet al. 2014), to investigate the effect of multitrophic biodiversity on stabilityrelated indices. Compared with polynomial, quadratic and exponential models in regression analysis, linear models fitted the data better and were employed to test the relationships between multitrophic biodiversity and stability-related indices.

We tested whether soil biodiversity affects interactions among plant functional groups by comparing responses of plant functional groups in the monocultures and species-mixed communities. In the monocultures, a mixedeffect model with harvest time point and plant species as random factors was employed to investigate the effect of soil biodiversity loss on shoot biomass of individual plant functional group. By excluding monocultures, we further tested the effect of soil biodiversity and plant species richness on shoot biomass of plant functional groups using a mixed-effect model with harvest time point as a random factor. These analyses were conducted by sub-setting data during wet and dry periods, separately. All data analyses were performed in the software $R 4.0 .0$ ( $R$-Core-Team 2020). For data handling, analysis and visualization, we used the packages dplyr , MASS ,nlme, ggplot2, patchwork, ggridges, reshape2 , AICcmodavg and cowplot. Details of data analysis can be found in the Supplementary Methods.

\section{Results}

Soil microbial diversity . The dilution-to-extinction approach was successful in creating a gradient of soil microbial (bacterial and fungal) diversity; importantly, soil microbial biomass, as indicated by respiration rates, was recovered after incubation (Fig. 2A, B and C; Table S2). Compared with the undiluted soil inocula, $57.91 \%$ of soil microbial taxa was lost through the $10^{-3}$ dilution, and $78.18 \%$ was lost during the following $10^{-6}$ dilution based on the number of soil microbial amplicon sequence variants (ASVs) (Fig. 2A). Low-abundance taxa, for instance, Chytridiomycota and Chlamydiae, were first eliminated during dilution (Fig. 2B and C). Although the microcosms were open to microbial re-colonization from the air, which increased soil bacterial diversity especially in the low soil biodiversity treatment, differences of fungal diversity were still observed among soil dilution treatments at the end of the experiment (Fig. S1 and Table S2). Besides, the diversity of fungal mutualists in the phylum of Glomeromycotawas dramatically decreased at the $10^{-3}$ dilution and was absent at the $10^{-6}$ dilution (Fig. S1A and Table S2). The diversity of plant fungal pathogens was deceased by soil dilution in the soil inocula, and was not altered at the last harvest (Table S2). Because soil biodiversity is dominated by soil microbes, soil microbial diversity was used as an indicator for soil biodiversity in the present study.

Temporal stability of plant community biomass production. Model selection with Akaike information criterion (AIC) suggests that the additive models including soil biodiversity (low, moderate, high) and plant diversity (both species and functional richness) are better than the corresponding models with the interaction term to explain all aspects of temporal stability (i.e., temporal stability, mean, standard deviation, asynchrony, and population variance) (Table S3 and S4). This indicates that soil biodiversity and plant diversity independently influenced stability-related indices (Fig. 3; Table S5 and S6). Soil biodiversity loss had consistent negative effects on the temporal stability of community biomass production and plant species asynchrony along a gradient of plant species richness, and plant species richness was positively associated with the temporal stability of community biomass production and plant species asynchrony (Fig. 3A and B). Soil biodiversity and plant species richness did not affect population variance (Fig. 3D). The temporal 
mean of community biomass production was positively related to plant species richness, while the standard deviation was negatively correlated with plant species richness (Fig. 3C and E). Soil biodiversity loss did not alter the temporal mean of community biomass production, while statistically significantly increasing the standard deviation (Fig. 3C and E). Asynchrony at both plant species and functional group level was positively related to the temporal stability of community biomass production, while soil biodiversity loss did not exert a significant effect on the asynchrony-stability relationship (Fig. S2). When monocultures were excluded from the analysis, plant species richness did not exert a significant effect on the temporal stability of community biomass production, although the effect of soil biodiversity was still observed (Table S5).

However, functional diversity in terms of functional richness increased the temporal stability of plant communities excluding monocultures (Fig. 3F). Plant species asynchrony, population variance and the temporal mean of community biomass production were increased by functional richness, and decreased by soil biodiversity loss (Fig. 3G, H and I). Soil biodiversity loss and functional richness did not alter the standard deviation of community biomass production (Fig. 3J). Furthermore, functional richness was closely related to plant species richness, and $78 \%$ of variance was explained by an additive model, which was much higher than that of other functional diversity indices $\left(R^{2}<40 \%\right.$, Table S6). Plant species richness did not explain functional evenness and the community-weighted mean of all traits (Table S6). Functional divergence and dispersion were increased by plant species richness, but were not correlated with the temporal stability (Table S7).

Multitrophic biodiversity was positively associated with the temporal stability of community biomass production, plant species asynchrony and the temporal mean of community biomass production, and negatively correlated with the standard deviation of community biomass production (Fig. 4A, B, C and E). Multitrophic biodiversity was not related to population variance (Fig. 4D). The linear relationships indicate that multitrophic biodiversity can drive the temporal stability of community biomass production through an increase in either soil biodiversity or plant species richness.

Plant community composition. Plant species loss and community evenness were affected by either soil biodiversity or plant species richness, but were not related to the temporal stability of community biomass production (Table S5). In the monocultures, soil biodiversity did not affect the temporal stability of grasses, while increasing the temporal stability of herbs and legumes (Fig. S3A, B and C). In mixedspecies communities, plant species richness increased the temporal stability of grasses, but did not affect the temporal stability of herbs and legumes (Fig. S3D, E and F). Moderate soil biodiversity increased the temporal stability of herbs, while soil biodiversity did not influence the temporal stability of grasses and legumes in mixed-species communities (Fig. S3D, E and F). Soil biodiversity loss increased the proportional abundance of grasses in mixed-species communities, but decreased the proportional abundance of herbs and legumes (Fig. S3G, H and I). The proportional abundance of grasses was positively associated with the temporal stability of community biomass production, and the proportional abundance of herbs and legumes was negatively associated with temporal stability (Fig. S3J, K and L). However, soil biodiversity loss still had negative effects on the temporal stability of community biomass production (Fig. S3J, K and L).

Soil biodiversity loss did not alter the shoot biomass of grasses and herbs during wet and dry periods in the monocultures (Table S8 and Fig. 5). Shoot biomass of legumes was increased by soil biodiversity loss during wet periods, and was not altered during dry periods in the monocultures. In mixed-species communities, the effects of soil biodiversity loss on shoot biomass of plant functional groups were similar along a gradient of plant species richness (Table S8 and Fig. 5). For instance, the loss of soil biodiversity increased the growth of grasses, while strongly decreasing the growth of herbs and legumes, independently of plant species richness. On average, there was at least twice as much shoot biomass of grasses as legumes or herbs at low soil biodiversity. Plant species richness did not affect shoot biomass of plant functional groups during wet periods, but tended to increase their shoot biomass during dry periods.

\section{Discussion}

Our findings show that plant and soil biodiversity have non-substitutable impacts on the temporal stability of community biomass production. In the present study, soil biodiversity loss had a detrimental effect 
on temporal stability by reducing the community-level mean biomass production, increasing the temporal variability, and weakening compensatory effects (via facilitation of uneven community composition favoring grasses over herbs and legumes). Consistent with previous studies (Tilman et al. 2006; Hector et al. 2010; Hautier et al. 2014; Hautier et al. 2015; Craven et al. 2018; Wanget al. 2019b), an increase in plant diversity in terms of both species richness and functional diversity promoted temporal stability. However, plant and soil biodiversity exerted independent effects on temporal stability. Furthermore, when multitrophic biodiversity was calculated from plant and soil biodiversity, multitrophic biodiversity was positively and linearly associated with the temporal stability of community biomass production. A large number of studies suggest that the biodiversity of a single trophic group, e.g. the diversity of plants, is a major factor stabilizing biomass production (Tilman et al.2006; Hector et al. 2010; Hautier et al. 2014; Hautier et al. 2015; Pennekamp et al. 2018; Craven et al.2018; Wang et al. 2019b). Our study suggests that maintaining ecosystem functions (e.g. biomass production) when faced with environmental variability requires biodiversity at multiple trophic levels.

Compared with plant species richness, the diversity of plant functional traits was a better explanatory variable for temporal stability in diverse plant communities. Exploitative plant species with fast-growing acquisitive traits, e.g. high specific leaf area, leaf nitrogen concentration, specific root length and specific root surface area, could recover rapidly following disturbance, while conservative species with slow-growing traits, e.g. low leaf dry matter concentration, high root average diameter and root tissue density, may have a higher resistance to disturbance (Fischeret al. 2016; Liu et al. 2018; Mahaut et al. 2020; Lozano et al. 2020; Freschet et al. 2020). Plant communities with a higher diversity of functional traits likely imply a stronger asynchronous response of plant species, e.g. a decrease in exploitative plant species compensated by conservative species during disturbance, and vice versa. In the present study, the diversity of plant functional traits increased plant species asynchrony, and subsequently, promoted the temporal stability of biomass production.

Plant species asynchrony is a main underlying mechanism by which plant diversity stabilizes biomass production when faced with disturbance (Loreau \& de Mazancourt 2008; Isbell et al. 2009; Hectoret al. 2010; de Mazancourt et al. 2013; Loreau \& de Mazancourt 2013; Thibaut \& Connolly 2013; Hautier et al. 2014; Craven et al. 2018; Zhang et al. 2019). Supporting past studies, we found that higher plant species richness, soil biodiversity and multitrophic biodiversity promoted the temporal stability of biomass production via enhancing plant species asynchrony. A previous study found that the presence of natural soil biota dramatically increased the growth of legumes and herbs, and thus, promoted plant species asynchrony under simulated environmental variation in precipitation (Pellkofer et al. 2016). Our results suggest that soil biodiversity is crucial for the occurrence of asynchrony of plant species and particularly functional groups. In accordance with past research (Wagg et al. 2014; Wagg et al. 2019; Prudentet al. 2020; Yang et al. 2020), we found that soil biodiversity enhanced the performance of herbs and legumes during wet and dry periods, likely indicating higher resistance during dry periods or faster recovery during wet periods. Specifically, herbs had the opportunity to recover from drought and differed from grasses in response to the simulated variation in precipitation at high soil biodiversity (Fig. 5). Besides, the dramatic reduction in legumes was compensated by the rapid growth of grasses during the first drought disturbance at high and moderate soil biodiversity. However, the compensatory effect was weaker in low soil biodiversity because legumes had already been suppressed by soil biodiversity loss before the first drought disturbance. A recent study shows that soil biodiversity is more important for herbs than grasses, and especially for the persistence of legumes (Yang et al. 2020). Besides, the growth of herbs and legumes is more reliable in the presence of mycorrhizal soil mutualists (Hoeksema et al. 2010; van der Heijden et al. 2016). The absence of these soil mutualists, in addition to the loss of soil biodiversity, could have suppressed the performance of herbs and legumes during and following drought in the present study.

It should be noted that soil biodiversity loss did not affect the growth of herbs and grasses, and even increased the growth of legumes in the monocultures. Therefore, soil biodiversity influenced the performance of plant functional groups by regulating interactions among functional groups in the mixed-species communities. For instance, soil biodiversity increased the mean of plant community evenness by favouring herbs and legumes. 
Although biodiversity can enhance the temporal stability by promoting community evenness (Thibaut \& Connolly 2013), we found that plant community evenness was not related to the temporal stability of community biomass production. Because the proportional abundance of grasses was positively related to temporal stability, soil biodiversity loss should increase temporal stability by promoting the dominance of grasses. However, soil biodiversity loss exerted a negative effect on temporal stability. Thus, the detrimental effect of soil biodiversity loss on temporal stability cannot be directly attributed to its effect on community evenness and the dominance of grasses, but can come from a decrease in asynchrony of plant functional groups.

A decrease in population variance can also contribute to the temporal stability of biomass production, because effects of treatments on temporal stability can be decomposed into their effects on plant species asynchrony and population variance (Thibaut \& Connolly 2013). Higher functional richness and soil biodiversity increased population variance, which should decrease temporal stability. However, these detrimental effects were neutralized by an increase in plant species asynchrony. An increase in the temporal stability of biomass production can be caused by both, an increase in the temporal mean of biomass production and a reduction in the temporal standard deviation (Tilman et al. 2006; Hautier et al. 2014). Consistent with most earlier studies (Tilman et al. 2014; Hautier et al. 2014; Craven et al. 2016; Weisser et al. 2017), our study supports the hypothesis that an increase in plant species richness can promote biomass production and decrease the temporal standard deviation of biomass production.

Soil biodiversity loss can decrease or increase biomass production, which depends on soil biodiversity and soil community composition (Wagg et al. 2014). Our results suggest that the temporal mean of biomass production was not altered by soil biodiversity loss when monocultures were included, while the temporal mean was decreased when monocultures were excluded (Fig. 3C and $\mathrm{H}$ ). This suggests that soil biodiversity loss has a detrimental effect on the temporal mean of biomass production in diverse plant communities. However, soil biodiversity loss did not affect plant diversity-productivity relationships in diverse plant communities. Past research shows soil pathogen suppression has more beneficial effects, while the absence of soil mutualists exerts stronger detrimental effects on biomass production in less diverse plant communities, and consequently, influences plant diversity-productivity relationships (Klironomos et al. 2000; Maron et al. 2011; Schnitzeret al. 2011; Luo et al. 2017; Liang et al. 2019; Wang et al. 2019a). However, soil biodiversity loss did not alter the strength of plant diversity effects on plant biomass production in the present study. These results indicate that the effect of soil biodiversity loss on plant productivity could not be simply due to the loss of single soil pathogens or mutualists. In the present study, soil biodiversity loss reduced plant fungal pathogens in soil inocula, and reduced or even eliminated mycorrhizal soil mutualists in the phylum of Glomeromycota at the last harvest. Reductions in soil pathogens and mutualists have opposite effects on plant biomass production, and therefore, effects of reductions in soil pathogens and mutualists along soil biodiversity loss might cancel each other out. A decrease in plant productivity could be caused by soil biodiversity loss in general other than the loss of soil mutualists along with soil biodiversity loss (Prudent et al. 2020; Yang et al. 2020).

Our study sheds new light on the consequences of multitrophic biodiversity loss under global anthropogenic change. Anthropogenic influences have been shown to destabilize biomass production via reducing plant diversity (Hautieret al. 2015). Moreover, global anthropogenic change, such as nitrogen deposition, land-use intensification, warming, fertilization and drought, can threaten both soil and plant biodiversity (Tsiafouliet al. 2015; Hautier et al. 2015; Gossner et al.2016; Banerjee et al. 2019; Geisen et al. 2019; Rilliget al. 2019; Zhou et al. 2020). Our study suggests that the loss of multitrophic biodiversity could reduce the temporal stability of biomass production by suppressing the occurrence of plant species asynchrony or decreasing the mean of biomass production and increasing its variation. These results indicate that the prediction of biomass production under global anthropogenic change requires a multitrophic evaluation of biodiversity loss.

In this study, the dilution-to-extinction approach, which has been widely used to investigate the relationships between rare soil microbial species and ecosystem functions (Yan et al. 2015; Hol et al. 2015; Roger et al. 2016; Maron et al. 2018; Domeignoz-Hortaet al. 2020), was employed to create a gradient of soil biodiversity. 
Our results confirm that less abundant taxa were lost first during dilution, followed by more abundant taxa (Yan et al.2015; Roger et al. 2016; Maron et al. 2018). This approach can simulate a realistic loss of soil biodiversity (Yanet al. 2015; Roger et al. 2016; Maron et al. 2018), because less abundant taxa have a higher risk of extinction than abundant species under anthropogenic changes (Zhou et al. 2020). The present study used an open system, and an increase in bacterial phyla was observed, compared to the initial soil inoculum. This means that propagules from the environment contributed to soil biodiversity at the last harvest. Contamination in a sterile treatment was even detected in closed systems (Wagg et al. 2014) and is generally difficult to avoid. Because soil biodiversity generally had a positive effect on ecosystem functions in previous studies and our present study, this indicates that we have underestimated the effect of soil biodiversity loss on the temporal stability of biomass production.

In summary, our study suggests that plant and soil biodiversity play non-substitutable roles in stabilizing plant community biomass production. Although greater plant diversity can promote the temporal stability of biomass production, the reduction in temporal stability induced by soil biodiversity loss cannot be compensated via increasing plant diversity. This result highlights the significance of multitrophic biodiversity for stabilizing ecosystem functions. Our study has important implications for restoration and conservation management in terrestrial ecosystems. First, it is important to preserve biodiversity at multiple trophic levels to stabilize ecosystem functions, especially in ecosystems suffering from intense disturbance. Second, although biomass production can be stabilized via increasing plant diversity by restoration or conservation measures, we should not ignore the importance of maintaining soil biodiversity, since low levels of soil biodiversity can destabilize biomass production.

\section{Acknowledgements}

We are grateful to Florine Degrune, James Whitehead, Max-Bernhard Ballhausen, Sabine Buchert, Anja Wulf, Bernd Richter, Helga Kanda, Carlos Aguilar, Yudi Lozano, Simone Weidner and Yun Liang for their assistance in laboratory and greenhouse work. The study was funded by the German science foundation (DFG Grant No. 434341960). M.R. acknowledges funding by the Grant-in-Aid for JSPS Overseas Research Fellowships.

\section{References}

Allan, E., Bossdorf, O., Dormann, C.F., Prati, D., Gossner, M.M., Tscharntke, T. et al. (2014). Interannual variation in land-use intensity enhances grassland multidiversity. Proc. Natl. Acad. Sci. U. S. A. , 111, 308-313.

Banerjee, S., Walder, F., Büchi, L., Meyer, M., Held, A.Y., Gattinger, A. et al. (2019). Agricultural intensification reduces microbial network complexity and the abundance of keystone taxa in roots.ISME J. , 13, 1722-1736.

Bardgett, R.D. \& van der Putten, W.H. (2014). Belowground biodiversity and ecosystem functioning. Nature , 515, 505-511.

Callahan, B.J., McMurdie, P.J., Rosen, M.J., Han, A.W., Johnson, A.J.A. \& Holmes, S.P. (2016). DADA2: High-resolution sample inference from Illumina amplicon data. Nat. Methods , 13, 581.

Craven, D., Isbell, F., Manning, P., Connolly, J., Bruelheide, H., Ebeling, A. et al. (2016). Plant diversity effects on grassland productivity are robust to both nutrient enrichment and drought.Philos. Trans. R. Soc. B-Biol. Sci. , 371, 20150277.

Craven, D., Eisenhauer, N., Pearse, W.D., Hautier, Y., Isbell, F., Roscher, C. et al. (2018). Multiple facets of biodiversity drive the diversity-stability relationship. Nat. Ecol. Evol. , 2, 1579-1587.

de Mazancourt, C., Isbell, F., Larocque, A., Berendse, F., De Luca, E., Grace, J.B. et al. (2013). Predicting ecosystem stability from community composition and biodiversity. Ecol. Lett. , 16, 617-625. 
Delgado-Baquerizo, M., Maestre, F.T., Reich, P.B., Jeffries, T.C., Gaitan, J.J., Encinar, D. et al. (2016). Microbial diversity drives multifunctionality in terrestrial ecosystems. Nat. Commun. , 7, 10541.

Domeignoz-Horta, L.A., Pold, G., Liu, X.J.A., Frey, S.D., Melillo, J.M. \& DeAngelis, K.M. (2020). Microbial diversity drives carbon use efficiency in a model soil. Nat. Commun., 11, 3684.

Fierer, N., Jackson, J.A., Vilgalys, R. \& Jackson, R.B. (2005). Assessment of soil microbial community structure by use of taxon-specific quantitative PCR assays. Appl. Environ. Microbiol. , 71, 4117-4120.

Fischer, F.M., Wright, A.J., Eisenhauer, N., Ebeling, A., Roscher, C., Wagg, C. et al. (2016). Plant species richness and functional traits affect community stability after a flood event. Philos. Trans. R. Soc. B-Biol. Sci. , 371, 20150276.

Freschet, G.T., Roumet, C., Comas, L.H., Weemstra, M., Bengough, A.G., Rewald, B. et al. (2020). Root traits as drivers of plant and ecosystem functioning: current understanding, pitfalls and future research needs. New Phytol. ,https://doi.org/10.1111/nph.17072.

Geisen, S., Wall, D.H. \& van der Putten, W.H. (2019). Challenges and opportunities for soil biodiversity in the Anthropocene. Curr. Biol., 29, R1036-R1044.

Gossner, M.M., Lewinsohn, T.M., Kahl, T., Grassein, F., Boch, S., Prati, D. et al. (2016). Land-use intensification causes multitrophic homogenization of grassland communities. Nature , 540, 266-269.

Guerra, C.A., Heintz-Buschart, A., Sikorski, J., Chatzinotas, A., Guerrero-Ramírez, N., Cesarz, S. et al. (2020). Blind spots in global soil biodiversity and ecosystem function research. Nat. Commun., 11, 3870.

Hautier, Y., Seabloom, E.W., Borer, E.T., Adler, P.B., Harpole, W.S., Hillebrand, H. et al. (2014). Eutrophication weakens stabilizing effects of diversity in natural grasslands. Nature , 508, 521-525.

Hautier, Y., Tilman, D., Isbell, F., Seabloom, E.W., Borer, E.T. \& Reich, P.B. (2015). Anthropogenic environmental changes affect ecosystem stability via biodiversity. Science, 348, 336-340.

Hector, A., Hautier, Y., Saner, P., Wacker, L., Bagchi, R., Joshi, J.et al. (2010). General stabilizing effects of plant diversity on grassland productivity through population asynchrony and overyielding.Ecology , 91, 2213-2220.

Hoeksema, J.D., Chaudhary, V.B., Gehring, C.A., Johnson, N.C., Karst, J., Koide, R.T. et al. (2010). A meta-analysis of context-dependency in plant response to inoculation with mycorrhizal fungi. Ecol. Lett. , 13, 394-407.

Hol, W.H.G., de Boer, W., de Hollander, M., Kuramae, E.E., Meisner, A. \& van der Putten, W.H. (2015). Context dependency and saturating effects of loss of rare soil microbes on plant productivity.Front. Plant Sci. , 6, 485 .

Ihrmark, K., Bödeker, I.T.M., Cruz-Martinez, K., Friberg, H., Kubartova, A., Schenck, J. et al. (2012). New primers to amplify the fungal ITS2 region - evaluation by 454-sequencing of artificial and natural communities. FEMS Microbiol. Ecol. , 82, 666-677.

Isbell, F.I., Polley, H.W. \& Wilsey, B.J. (2009). Biodiversity, productivity and the temporal stability of productivity: patterns and processes. Ecol. Lett. , 12, 443-451.

Klironomos, J.N., McCune, J., Hart, M. \& Neville, J. (2000). The influence of arbuscular mycorrhizae on the relationship between plant diversity and productivity. Ecol. Lett. , 3, 137-141.

Laliberté, E. \& Legendre, P. (2010). A distance-based framework for measuring functional diversity from multiple traits. Ecology, 91, 299-305.

Lê, S., Josse, J. \& Husson, F. (2008). FactoMineR : an R package for multivariate analysis. 2008 , 25, 18. 
Liang, M., Liu, X., Parker, I.M., Johnson, D., Zheng, Y., Luo, S.et al. (2019). Soil microbes drive phylogenetic diversity-productivity relationships in a subtropical forest. Sci Adv , 5, eaax5088.

Liu, H., Mi, Z., Lin, L., Wang, Y., Zhang, Z., Zhang, F. et al.(2018). Shifting plant species composition in response to climate change stabilizes grassland primary production. Proc. Natl. Acad. Sci. U. S. A., 115, 4051-4056.

Loreau, M. \& de Mazancourt, C. (2008). Species synchrony and its drivers: neutral and nonneutral community dynamics in fluctuating environments. Am. Nat. , 172, E48-E66.

Loreau, M. \& de Mazancourt, C. (2013). Biodiversity and ecosystem stability: a synthesis of underlying mechanisms. Ecol. Lett. , 16, 106-115.

Lozano, Y.M., Aguilar-Trigueros, C.A., Flaig, I.C. \& Rillig, M.C. (2020). Root trait responses to drought are more heterogeneous than leaf trait responses. Funct. Ecol. , 34, 2224-2235.

Lozano, Y.M. \& Rillig, M.C. (2020). Effects of Microplastic Fibers and Drought on Plant Communities. Environ. Sci. Technol. , 54, 6166-6173.

Luo, S., De Deyn, G.B., Jiang, B. \& Yu, S. (2017). Soil biota suppress positive plant diversity effects on productivity at high but not low soil fertility. J. Ecol. , 105, 1766-1774.

Ma, Z., Liu, H., Mi, Z., Zhang, Z., Wang, Y., Xu, W. et al.(2017). Climate warming reduces the temporal stability of plant community biomass production. Nat. Commun., 8, 15378.

Mahaut, L., Fort, F., Violle, C. \& Freschet, G.T. (2020). Multiple facets of diversity effects on plant productivity: species richness, functional diversity, species identity and intraspecific competition.Funct. Ecol. , 34, 287-298.

Maron, J.L., Marler, M., Klironomos, J.N. \& Cleveland, C.C. (2011). Soil fungal pathogens and the relationship between plant diversity and productivity. Ecol. Lett. , 14, 36-41.

Maron, P.A., Sarr, A., Kaisermann, A., Leveque, J., Mathieu, O., Guigue, J. et al. (2018). High microbial diversity promotes soil ecosystem functioning. Appl. Environ. Microbiol., 84.

Orgiazzi, A., Bardgett, R.D. \& Barrios, E. (2016). Global soil biodiversity atlas . European Commission, Luxembourg.

Pellkofer, S., van der Heijden, M.G.A., Schmid, B. \& Wagg, C. (2016). Soil communities promote temporal stability and species asynchrony in experimental grassland communities. Plos One, 11, e0148015.

Pennekamp, F., Pontarp, M., Tabi, A., Altermatt, F., Alther, R., Choffat, Y. et al. (2018). Biodiversity increases and decreases ecosystem stability. Nature, 563, 109-112.

Prudent, M., Dequiedt, S., Sorin, C., Girodet, S., Nowak, V., Duc, G.et al. (2020). The diversity of soil microbial communities matters when legumes face drought. Plant Cell Environ. , 43, 1023-1035.

$R$-Core-Team (2020). $R$ : a language and environment for statistical computing. $R$ Foundation for Statistical Computing, Vienna, Austria.

Rillig, M.C., Mardatin, N.F., Leifheit, E.F. \& Antunes, P.M. (2010). Mycelium of arbuscular mycorrhizal fungi increases soil water repellency and is sufficient to maintain water-stable soil aggregates. Soil Biol. Biochem. , 42, 1189-1191.

Rillig, M.C., Ryo, M., Lehmann, A., Aguilar-Trigueros, C.A., Buchert, S., Wulf, A. et al. (2019). The role of multiple global change factors in driving soil functions and microbial biodiversity.Science, 366, 886-890.

Roger, F., Bertilsson, S., Langenheder, S., Osman, O.A. \& Gamfeldt, L. (2016). Effects of multiple dimensions of bacterial diversity on functioning, stability and multifunctionality. Ecology, 97, 2716-2728. 
Schnitzer, S.A., Klironomos, J.N., HilleRisLambers, J., Kinkel, L.L., Reich, P.B., Xiao, K. et al. (2011). Soil microbes drive the classic plant diversity-productivity pattern. Ecology , 92, 296-303.

Schuldt, A., Assmann, T., Brezzi, M., Buscot, F., Eichenberg, D., Gutknecht, J. et al. (2018). Biodiversity across trophic levels drives multifunctionality in highly diverse forests. Nat. Commun. , 9, 2989.

Soliveres, S., van der Plas, F., Manning, P., Prati, D., Gossner, M.M., Renner, S.C. et al. (2016). Biodiversity at multiple trophic levels is needed for ecosystem multifunctionality. Nature, 536, 456-459.

Song, M.-H. \& Yu, F.-H. (2015). Reduced compensatory effects explain the nitrogen-mediated reduction in stability of an alpine meadow on the Tibetan Plateau. New Phytol. , 207, 70-77.

Thakur, M.P., Phillips, H.R.P., Brose, U., De Vries, F.T., Lavelle, P., Loreau, M. et al. (2020). Towards an integrative understanding of soil biodiversity. Biological Reviews , 95, 350-364.

Thibaut, L.M. \& Connolly, S.R. (2013). Understanding diversity-stability relationships: towards a unified model of portfolio effects. Ecol. Lett. , 16, 140-150.

Tilman, D. \& Downing, J.A. (1994). Biodiversity and stability in grasslands. Nature, 367, 363-365.

Tilman, D., Reich, P.B. \& Knops, J.M.H. (2006). Biodiversity and ecosystem stability in a decade-long grassland experiment.Nature , 441, 629-632.

Tilman, D., Isbell, F. \& Cowles, J.M. (2014). Biodiversity and ecosystem functioning. Annu. Rev. Ecol. Evol. Syst. , 45, 471-493.

Tsiafouli, M.A., Thébault, E., Sgardelis, S.P., de Ruiter, P.C., van der Putten, W.H., Birkhofer, K. et al. (2015). Intensive agriculture reduces soil biodiversity across Europe. Glob. Change Biol., 21, 973-985.

van der Heijden, M.G.A., Bruin, S.d., Luckerhoff, L., van Logtestijn, R.S.P. \& Schlaeppi, K. (2016). A widespread plant-fungal-bacterial symbiosis promotes plant biodiversity, plant nutrition and seedling recruitment. ISME J. , 10, 389-399.

Villéger, S., Mason, N.W.H. \& Mouillot, D. (2008). New multidimensional functional diversity indices for a multifaced framework in functional ecology. Ecology, 89, 2290-2301.

Wagg, C., Bender, S.F., Widmer, F. \& van der Heijden, M.G.A. (2014). Soil biodiversity and soil community composition determine ecosystem multifunctionality. Proc. Natl. Acad. Sci. U. S. A., 111, 5266-5270.

Wagg, C., Schlaeppi, K., Banerjee, S., Kuramae, E.E. \& van der Heijden, M.G.A. (2019). Fungal-bacterial diversity and microbiome complexity predict ecosystem functioning. Nat. Commun., 10, 4841.

Wang, G.Z., Schultz, P., Tipton, A., Zhang, J.L., Zhang, F.S. \& Bever, J.D. (2019a). Soil microbiome mediates positive plant diversity-productivity relationships in late successional grassland species. Ecol. Lett. , 22, 1221-1232.

Wang, Y., Cadotte, M.W., Chen, Y., Fraser, L.H., Zhang, Y., Huang, F.et al. (2019b). Global evidence of positive biodiversity effects on spatial ecosystem stability in natural grasslands. Nat. Commun., 10, 3207.

Weisser, W.W., Roscher, C., Meyer, S.T., Ebeling, A., Luo, G.J., Allan, E. et al. (2017). Biodiversity effects on ecosystem functioning in a 15-year grassland experiment: Patterns, mechanisms, and open questions. Basic Appl. Ecol. , 23, 1-73.

Yan, Y., Kuramae, E.E., Klinkhamer, P.G.L. \& van Veen, J.A. (2015). Revisiting the dilution procedure used to manipulate microbial biodiversity in terrestrial systems. Appl. Environ. Microbiol. , 81, 4246-4252.

Yang, G., Wagg, C., Veresoglou, S.D., Hempel, S. \& Rillig, M.C. (2018). How soil biota drive ecosystem stability. Trends Plant Sci. , 23, 1057-1067.

Yang, G., Roy, J., Veresoglou, S.D. \& Rillig, M.C. (2020). Soil biodiversity enhances the persistence of legumes under climate change.New Phytol., 10.1111/nph.17065. 
Zhang, Y., Feng, J., Loreau, M., He, N., Han, X. \& Jiang, L. (2019). Nitrogen addition does not reduce the role of spatial asynchrony in stabilising grassland communities. Ecol. Lett. , 22, 563-571.

Zhou, Z., Wang, C. \& Luo, Y. (2020). Meta-analysis of the impacts of global change factors on soil microbial diversity and functionality.Nat. Commun., 11, 3072.

\section{Figure legends}

Figure 1 . Scheme of the experimental design. (1)Dilution. Fresh field soil was kept undiluted or diluted $1 \times 10^{3}$ and $1 \times 10^{6}$ times using sterilized soil to create high, moderate and low soil biodiversity inocula, respectively. (2) Incubation . Each soil bag was sealed using a sterilized cotton plug to avoid aerial microbial contamination while permitting gas exchange, and then incubated at room temperature in the dark until similar microbial abundance was observed among dilution treatments. (3) Soil inoculation and transplanting. After incubation, $200 \mathrm{~g}$ of soil inoculum was homogenized with $6800 \mathrm{~g}$ of sterilized soil and sand mixture (1:1) in each microcosm. Each microcosm received 24 seedlings from 1, 4, 8 or 12 plant species depending on plant diversity treatment. (4) Simulating environmental variation in precipitation . Three wet-dry cycles were implemented by maintaining gravimetric soil moisture of $12-18 \%$ during the wetting periods and by watering until most plants started to wilt during the drying periods. At the end of each period, plant shoots were cut at $5 \mathrm{~cm}$ above the soil surface to determine shoot biomass production.

Figure 2. The effect of the dilution-to-extinction approach on soil microbial diversity, biomass (indicated by soil respiration rate after incubation) and community composition. (A) The dilution-to-extinction approach successfully reduced soil microbial diversity (solid line) but did not alter the soil respiration rate (dash line). The dilution-to-extinction approach dramatically reduced fungal (B) and bacterial (C) diversity and altered the community composition of soil microbes based on amplicon sequence variant (ASV) detection. Error bar represents standard deviation $(\mathrm{n}=3)$.

Figure 3. Soil biodiversity and plant diversity independently influence stability-related indices. (A - E) The effects of plant species richness (PS) and soil biodiversity on the temporal stability, mean and standard deviation of community biomass production, and plant species asynchrony and population variance. (F - J) The effects of plant functional richness and soil biodiversity loss on these stability-related indices. Effects of treatments on temporal stability were partitioned into effects on plant species asynchrony and population variance, or effects on temporal mean and standard deviation of community biomass production. Light bands represent $95 \%$ confidence intervals. Note: moderate and low soil biodiversity effects refer to the differences to the high soil biodiversity treatment. ${ }^{* *} P<0.001 ;{ }^{* *} P<0.01{ }^{*} P<0.05$; ns $P>0.05$.

Figure 4.Relationships between multitrophic biodiversity and (A) the temporal stability of community biomass production, (B) plant species asynchrony, (D) population variance, (C) the temporal mean and (E) standard deviation of community biomass production. Effects of treatments on temporal stability were partitioned into effects on plant species asynchrony and population variance, or effects on temporal mean and standard deviation of community biomass production. Light bands represent $95 \%$ confidence intervals.

Figure 5 . Shoot biomass of grasses, herbs and legumes at each harvest when microcosms were inoculated with high, moderate or low soil biodiversity inoculum in the plant communities with $1,4,8$ or 12 species. 

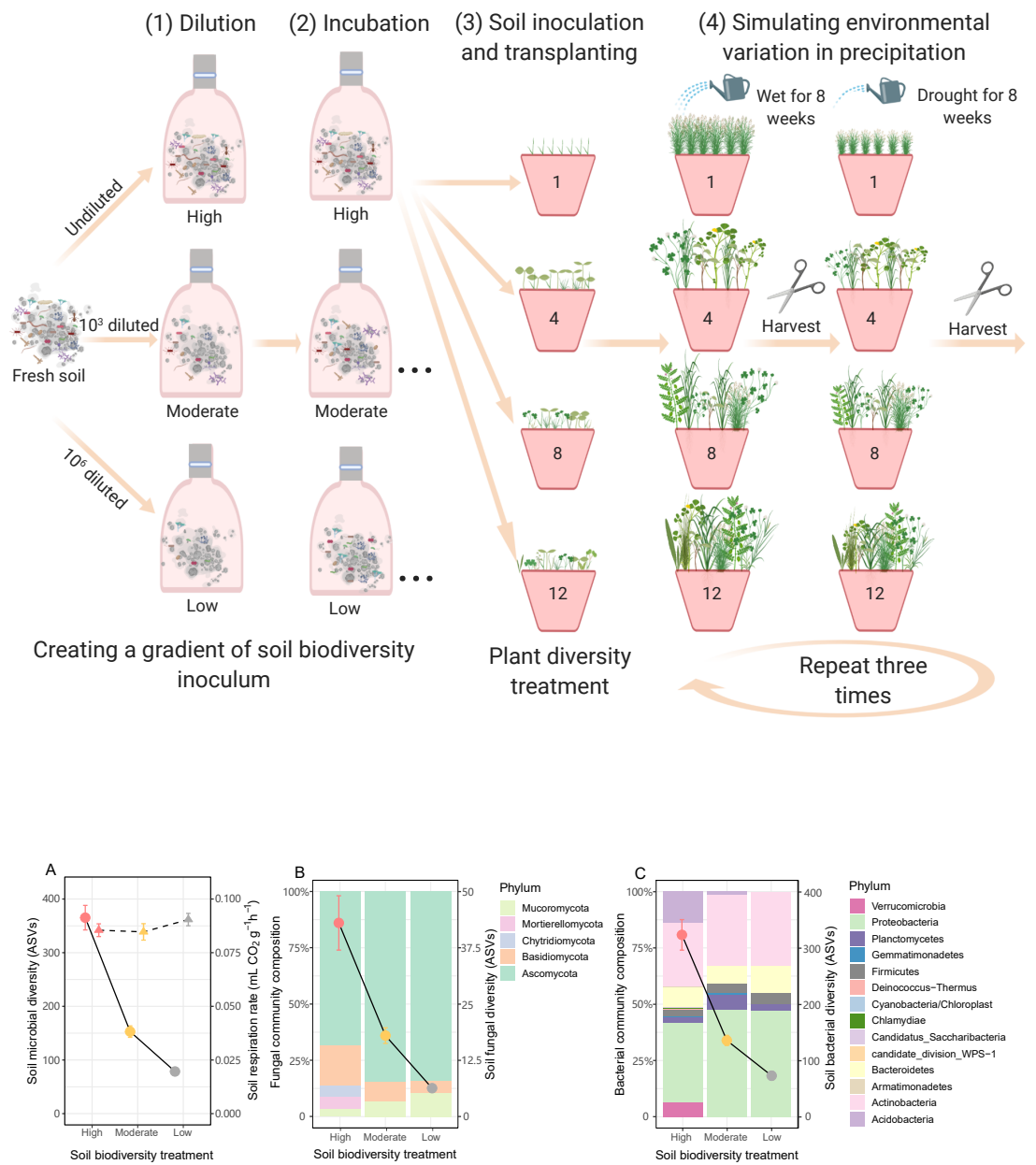


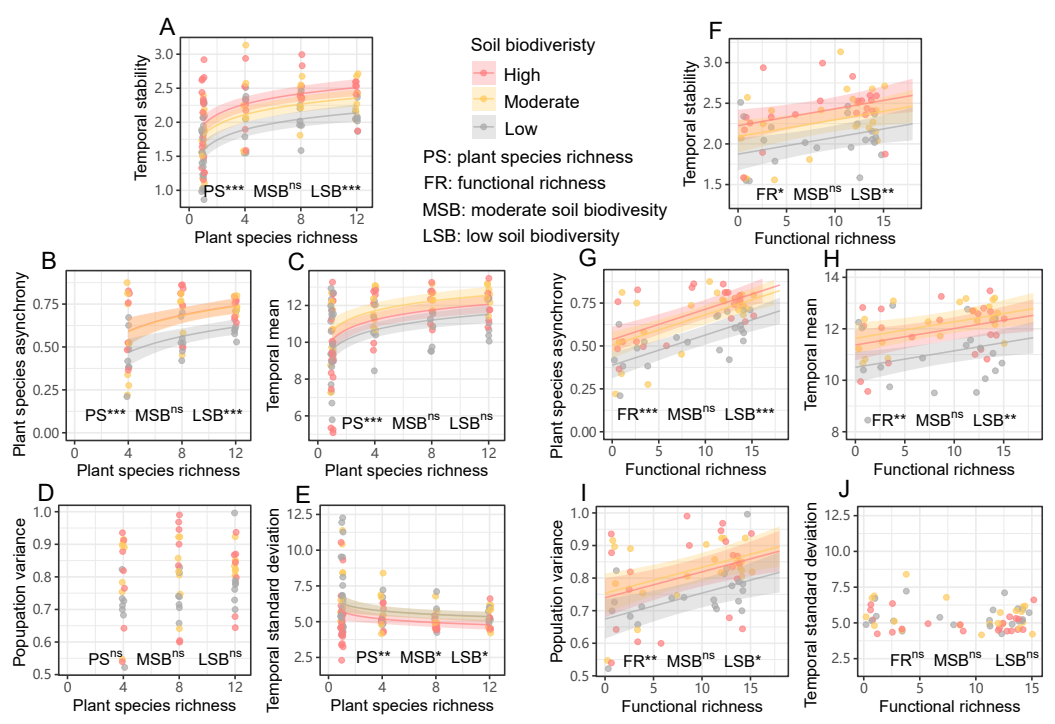



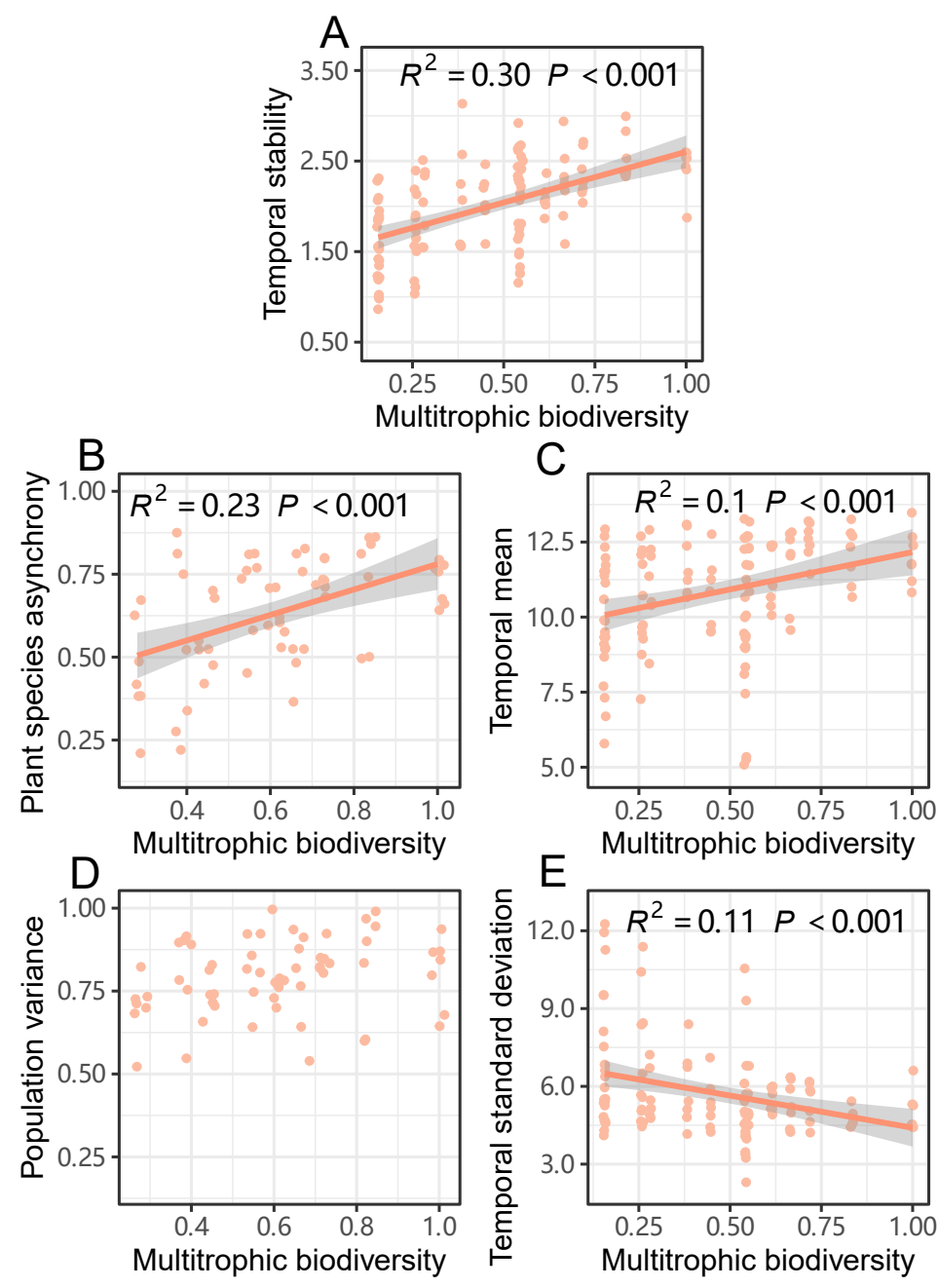


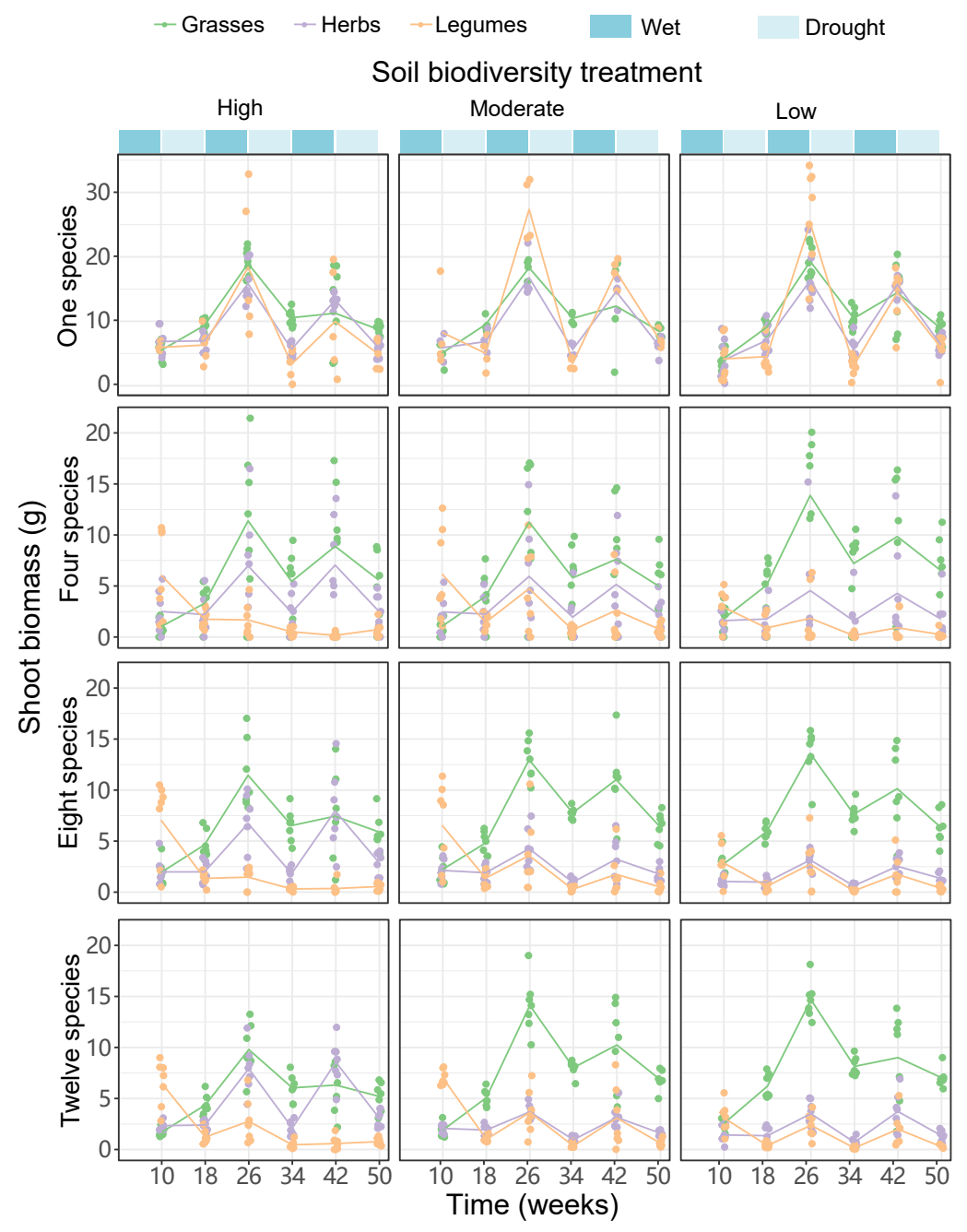

